\title{
Global Surface Parameterization by Smooth Facet Selection
}

\author{
Ralf Vogelgesang \\ Max Planck Institute for Solid State Research, 70569 Stuttgart, Germany
}

\begin{abstract}
We present a global surface parameterization for smooth, star-like domains with asymptotic singularities (edges and corners) whose "sharpness" can be tuned. The basic idea is a novel utilization of the (near-)maximum norm. For a given parameterized direction, by choosing the largest inverse distance, it "selects" out of a set of limiting facets the one which is closest. Thus each facet's orientation and location can be individually controlled. Assembly of the final surface happens automatically and $C^{\infty}$-smoothly over the entire parameter domain, i.e., the unit circle (2D) or unit sphere (3D). The resulting surface is directly amenable to fast Fourier transforms and related methods and should also be useful for many high-order numerical solvers. We demonstrate how to construct general convex polygons and polyhedra and point out a few possibilities for concave, star-like domains.
\end{abstract}

\section{Keywords:}

\section{INTRODUCTION}

This research was inspired by the tremendously growing variety of methods to fabricate plasmonic nanoparticles ${ }^{1-3}$ as well as recent advances in measuring ${ }^{4-8}$ and calculating their properties with the help of boundary discretization methods. ${ }^{9-14}$ These metallic structures are typically several tens to hundreds of nanometers in extent and exhibit strong electromagnetic resonances in the visible to infrared frequency spectrum. Their optical properties are widely tunable by controlling the particle size and shape (see Fig. 1). In colloid-chemical growth methods, their shape and geometry is often driven by internal crystallographic symmetry, giving rise to, e.g., cubic, tetrahedral, octahedral, cuboctahedral, even dodecahedral and icosahedral particle shapes. These may be extended further into platelets or rods of triangular, rectangular, pentagonal, or hexagonal cross sections. This list does not exhaust the morphological possibilities, though. Specific thermo-chemical treatment processes allow rounding or sharpening of edges and corners, site-selective chemistry allows outward growth of rods ("legs") from a central particle, resulting in star-like structures. Further variety stems from the possibilities of heterogeneous growth of structures, using different materials, resulting, for instance, in core-shell structures or segmented rods. In the following, we present an intuitive, yet general scheme that allows modelling most of these morphologies of nano-particles.

Among the methods and algorithms available for numerical solutions of scattering problems in piece-wise homogeneous media, interface or boundary discretization methods are particularly efficient, largely due to the reduced dimensionality of the problem. ${ }^{15-17}$ For three(two-) dimensional objects only a two- (one-) dimensional manifold has to be considered. This results in low memory usage even for scattering objects much larger than one wavelength. Boundary integral equation methods, ${ }^{18}$ in conjunction with low operation count methods such as Fast Fourier Transforms (FFT), render the differential equations accurately solvable with high-order convergence even on modern desktop computers. This class of numerical problems has in general two sub-parts. First, a suitable geometric parameterization has to be found for the interface surfaces separating the different spatial domains. Second, an appropriate representation on these interfaces is required for the partial fields and/or potentials, which are then subject to the numerical solution process. In this report, we focus on the first part exclusively.

Most general approaches to the construction of interfaces are based on meshing routines that approximate the surface by patches of elemental geometry (often triangles). While extremely flexible, these schemes required considerable effort not only in book-keeping of issues such as connectivity of the patches, and in ensuring continuity of global functions, such as potentials, surface shape, etc. Often they are defined locally on each patch, and special conditions must be met between neighbouring patches. To keep computation costs manageable, the resulting representations of mesh-parameterized surfaces are usually of limited continuity, say continuous first-order derivatives, 


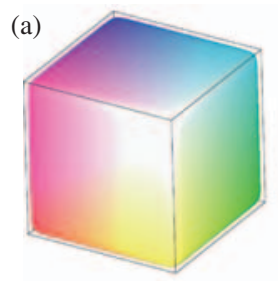

(e)

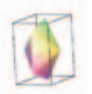

(i)

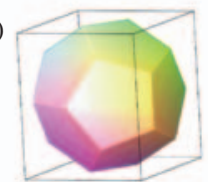

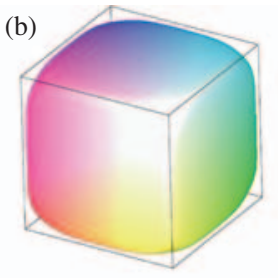

(f)

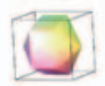

(j)

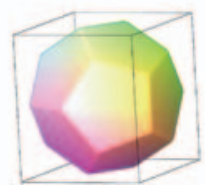

(c)

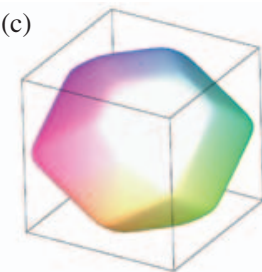

(g)

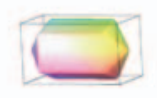

(k)

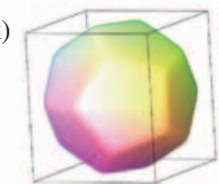

(d)

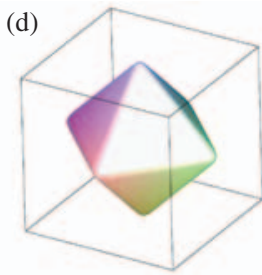

(h)

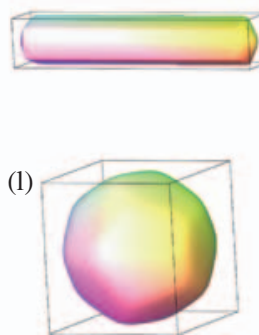

Fig. 1. Illustrations of typical shape-changing processes in nano-particle fabrication and the corresponding variations to particle surface geometry. (a-d) In cubic crystals, the ratio of rates of growth perpendicular to the $\{100\}$ and $\{111\}$ facets determines whether the resultant particle shape is a cube (a), an octahedron (d), or an intermediate case (b, c). (e-h) Growth of a pentagonal rod (h) occurs after stabilization of $\{100\}$ facets in a multiply twinned seed crystal (e). (i-l) Thermal annealing may result in rounding of corners and edges. All of these examples have been rendered with the representation of Eqs. (6), (7).

but discontinuous curvature. This limits their applicability to high-order numerical methods for boundary value problems. Suitable surface representations for use with high-order numerical methods for boundary value problems (such as partial differential equation solvers, integral equation solvers, etc.) require higher order continuity. ${ }^{19}$ Large area patches have been suggested based on Fourier analysis to continue smooth portions of piecewise smooth functions, which can thus cover extremely complex surface geometries. ${ }^{20}$ Our present goal is less ambitious, in that we deal with surfaces that can be considered radial deformations of a single unit circle or unit sphere. The advantage of this restriction is direct applicability of fast methods like FFT on the unit circle and Fast Spherical Harmonics Transform (FSHT) on the sphere. ${ }^{21-23}$

\section{BASIC DESCRIPTION OF SURFACE PARAMETERIZATION BY SMOOTH FACET SELECTION}

The starting point of our surface representation is the wellknown $p$-norm for finite-dimensional vector spaces,

$$
\|\mathbf{v}\|_{p}=\left(\sum_{i=1}^{N}\left|v_{i}\right|^{p}\right)^{1 / p}
$$

where $\mathbf{v}$ is an element of an $N$-dimensional vector space and $p>0$ a real number. One may consider the $p$-norm as a convenient way to smoothly parameterize corners.

As is illustrated in Figure 2(a), for higher values of $p$, the iso-norm curves of $p$-length 1.0 asymptotically approach the shape of a square. Indeed, for $p \rightarrow \infty$, the infinity-norm is also referred to as the maximum norm, since it selects the largest of the values $\left\{\left|v_{i}\right|\right\}$. This draws our attention on the unit vector pointing in the direction of the associated largest vector component (Fig. 2(b)). Viewed as an asymptotic limit, the iso-norm curve of the maximum norm can be viewed as composed of hypersurface segments. From Eq. (1), these are given implicitly by

$$
v_{i}=\mathbf{v} \cdot \mathbf{e}_{i}=1
$$

which may be generalized to

$$
\frac{[\mathbf{v}-\mathbf{R}] \cdot \mathbf{e}_{i}}{d_{i}}=1
$$

Here $d_{i}$ is the distance of the $i$-th surface segment from the origin $\mathbf{R}$. At this point we dissociate the index $i$ from the coordinate axes and let it become an index over an
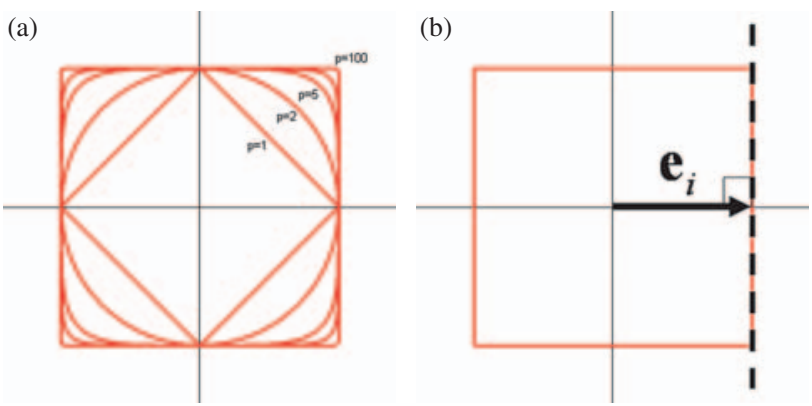

Fig. 2. (a) $\|\mathbf{v}\|_{p}=1$ iso-norm traces in $R^{2}$ implicitly defined by Eq. (1), for different values of $p$. (b) The "infinity" or "maximum" norm isocurve, viewed as composed of hyper-surface segments. The dashed line indicates the hyper-surface which constitutes one such segment-defined here as all points in the plane normal to $\mathrm{e}_{i}$ that has unit distance from the origin. 
arbitrary number of facets in the asymptotic surfaces. The coordinate parameterization is conveniently recovered by cylindrical or spherical angle coordinates in two- or threedimensional space, respectively, which at the same time become the surface coordinate parameters. For a given set of surface parameters $(\theta, \phi)$, we impose Eq. (1) as the determinant for the length $r$ of the vector reaching from $\mathbf{R}$ to $\mathbf{v}$ on the asymptotic surface. Let $\Delta(\theta, \phi)$ be the unit vector corresponding to the parameters $\theta$ and $\phi$. Usually, one will choose the standard cylindrical or spherical directions, $\boldsymbol{\Delta}(\phi)=\left(\cos (\phi) \mathbf{e}_{x}+\sin (\phi) \mathbf{e}_{y}\right)$ or $\boldsymbol{\Delta}(\theta, \phi)=$ $\left(\sin (\theta) \cos (\phi) \mathbf{e}_{x}+\sin (\theta) \sin (\phi) \mathbf{e}_{y}+\cos (\theta) \mathbf{e}_{z}\right)$, in a static reference frame. It may be convenient, though, to allow for a continuously variable reference frame, which then implies correspondingly varying Cartesian components for this unit vector. Using this kind of a parameterization, we can re-write the generalized Eq. (1) as

$$
\|\Delta(\theta, \phi)\|_{p}=1=\left(\sum_{i=1}^{N}\left|\frac{r \Delta(\theta, \phi) \cdot \mathbf{e}_{i}}{d_{i}}\right|^{p}\right)^{1 / p}
$$

That is, with

$$
r(\theta, \phi)=\left(\sum_{i=1}^{N}\left|\frac{\Delta(\theta, \phi) \cdot \mathbf{e}_{i}}{d_{i}}\right|^{p}\right)^{-1 / p}
$$

we can specify the location on the surface associated with $(\theta, \phi)$ as

$$
\mathbf{v}(\theta, \phi)=\mathbf{R}+r(\theta, \phi) \Delta(\theta, \phi)
$$

Nevertheless, Eq. (5) is unsatisfactory in one critical respect: The necessary use of absolute values implies the loss of a sign. The resulting curves exhibit an undesired "inversion symmetry." This is exemplified in Figure 3 for the case of a triangular asymptotic curve. Here the origin is $\mathbf{R}=\mathbf{0}$. When $r(\phi)$ is evaluated according to Eq. (5), one obtains a hexagon rather than a triangle, because any distinction between hyper-surfaces of distance $d_{i}$ and $-d_{i}$ is lost. This has to be re-established explicitly by a modification of Eq. (5), such as,

$$
r(\theta, \phi)=\left(\sum_{i=1}^{N} H\left(\frac{\Delta(\theta, \phi) \cdot \mathbf{e}_{i}}{d_{i}}\right)\left|\frac{\Delta(\theta, \phi) \cdot \mathbf{e}_{i}}{d_{i}}\right|^{p}\right)^{-1 / p}
$$
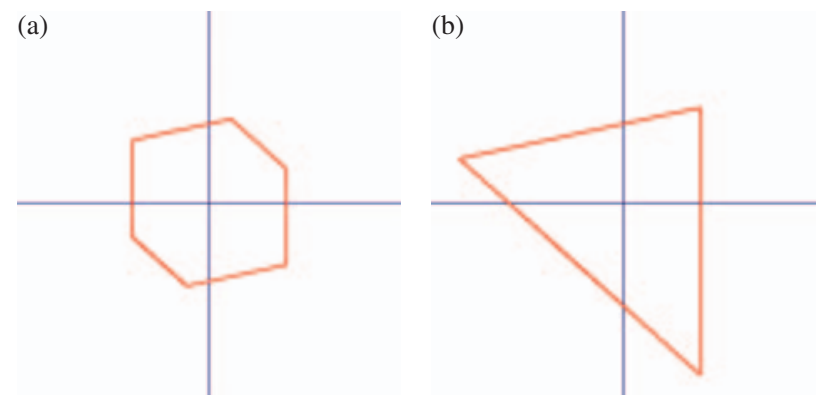

Fig. 3. The symmetry effect of using absolute values in the $p$-norm. (a) An iso-infinity-norm curve for three linear facets, using Eq. (5). (b) The same as in (a), but using Eq. (7). where the Heaviside step function $H(x)$ is zero for negative $x$ and one otherwise. Using Eq. (7), one obtains the desired triangle (Fig. 3(b)), as expected. Together, Eqs. (7) and (6) are the central result of this report. They have been used for generating all subpanels of Figure 1. For example, the dodecahedron of Figure 1(i-1) is created with the following set:

$$
\begin{gathered}
\alpha=2 \arctan \left(\frac{1+\sqrt{5}}{2}\right) \\
\theta_{i}=\{0, \alpha, \alpha, \alpha, \alpha, \alpha, \pi-\alpha, \pi-\alpha, \pi-\alpha, \pi-\alpha, \pi-\alpha, \pi\} \\
\phi_{i}=\left\{0, \frac{0}{5} \pi, \frac{2}{5} \pi, \frac{4}{5} \pi, \frac{6}{5} \pi, \frac{8}{5} \pi, \frac{1}{5} \pi, \frac{3}{5} \pi,\right. \\
\left.\frac{5}{5} \pi, \frac{7}{5} \pi, \frac{9}{5} \pi, 0\right\} \\
\mathbf{e}_{i}=\Delta\left(\theta_{i}, \phi_{i}\right)=\sin \left(\theta_{i}\right) \cos \left(\phi_{i}\right) \mathbf{e}_{x} \\
+\sin \left(\theta_{i}\right) \sin \left(\phi_{i}\right) \mathbf{e}_{y}+\cos \left(\theta_{i}\right) \mathbf{e}_{z} \\
d_{i}=\{1,1,1,1,1,1,1,1,1,1,1,1\}
\end{gathered}
$$

\section{CHARACTERISTICS AND EXPANSIONS}

\subsection{The Smallest Convex Enclosure Principle}

Next we discuss some of the features of surface parameterizations using Eqs. $(6,7)$. For clarity, we only display two-dimensional cases here. Three-dimensional generalizations are straightforward. Foremost, one realizes that the resulting surface may be construed as the smallest convex enclosure of the origin. This principle is illustrated in Figure 4. For each pair of direction vector and distance, one can envision the corresponding line (or plane in three dimensions) drawn. As the surface parameterization uses cylindrical (spherical) angles, it "tests" all directions around the origin. The generalized $p$-norm selects for each direction the reference line (plane) closest.

One may arbitrarily shift (altering $d_{i}$ ) and rotate (altering $\mathbf{e}_{i}$ ) the reference lines to morph the resulting enclosure
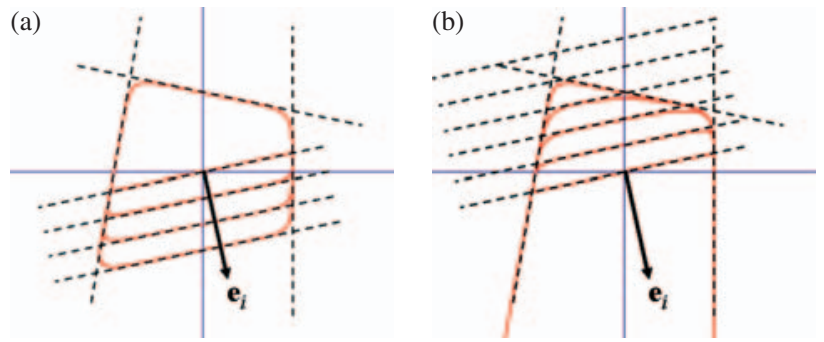

Fig. 4. Illustration of the smallest convex enclosure principle. A set of four lines (dotted) is considered. Each line is defined by its normal direction $\mathbf{e}_{i}$ and its distance $d_{i}$ from the origin. The red curve is generated by Eqs. $(6,7)$ with a modest rounding of corners $(p=9)$. (a) the distance of one line ist reduced to near-zero. (b) Continued reduction of the same distance as in (a) to negative values. 
curve to any desired shape (Fig. 4(a)). Changing the sign of a distance $d_{i}$ is equivalent to inverting the orientation $\mathbf{e}_{i}$-with corresponding drastic changes (Fig. 4(b)).

Note that under certain conditions, the "enclosing curve" may be open, like in Figure 4(b). This happens, whenever for a given $\phi$ (and $\theta$ ), all the conditionals introduced in Eq. (7) vanish,

$$
\forall i: H\left(\frac{\Delta(\theta, \phi) \cdot \mathbf{e}_{i}}{d_{i}}\right)=0
$$

and there are "no terms to choose from" for the $p$-norm. Also note that a certain line (plane) may lie completely outside the smallest convex enclosure, which is entirely composed of the other lines (planes), as is the case, e.g., for the most negative distance line displayed in Figure 4(b). Under such circumstances this line does not contribute anything to the limiting morphology $(p \rightarrow \infty)$, and only a small effect is noticeable for finite $p$.

The notion suggested by the limiting "maximum" norm (for $p \rightarrow \infty$ ) that a certain (the closest) facet is picked out of all available can be utilized further, to construct also concave forms. The main idea is that certain facets are only offered in certain parameter intervals. Algebraically, this is facilitated by additional conditionals, say

$$
\begin{aligned}
r(\theta, \phi)= & \left(\sum_{i=1}^{N} H\left(\phi-\phi_{\min , i}\right) H\left(\phi_{\max , i}-\phi\right) H\right. \\
& \left.\times\left(\frac{\Delta(\theta, \phi) \cdot \mathbf{e}_{i}}{d_{i}}\right)\left|\frac{\Delta(\theta, \phi) \cdot \mathbf{e}_{i}}{d_{i}}\right|^{p}\right)^{-1 / p}
\end{aligned}
$$

where $\phi_{\min , i}$ and $\phi_{\max , i}$ are the limits of the intervals of validity for facet $i$. Figure 5 shows an example. For more complicated situations, the determination of proper parameter intervals, however, is not that straightforward, and control of the roundedness of concave corners is also awkward.

\subsection{Controlling Local Surface Properties}

An interesting consequence of the opportunity to shift the defining lines (planes) is the possibility to re-locate the entire the structure on a different origin, by redefining

$$
d_{i} \rightarrow d_{i}+\mathbf{\Delta} \mathbf{R} \cdot \mathbf{e}_{i}
$$
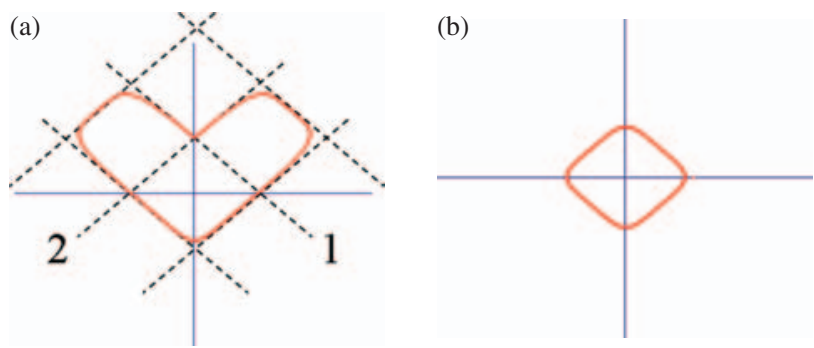

Fig. 5. Extension of the smallest convex enclosure principle by conditional faceting. In (a), facets 1 and 2 enter Eq. (9) only when parameter $\phi$ is in the interval $[\pi / 2,3 \pi / 2)$ and $[0, \pi / 2$ ), respectively. (b) shows the result of unconditional inclusion of all facets for all parameter values. as illustrated in Figure 6(a). For numerical considerations such a global shift may be useful to manage the parameter density along the circumference of the resulting shape. Of course, in view of the previous comments, care should be taken that none of the distances switch sign by this reassignment to preserve the resulting shape.

Let us emphasize the view that the angles $\phi$ and $\theta$ are mere parameters. For example, one may parameterize the global sharpness $p$ to become a local function $p(\phi)$, affording us options to increase it locally in the vicinity of a particular corner by a convenient function such as

$$
p \rightarrow p(\phi)=p_{0}+p_{c} e^{-\left(\left(\phi-\phi_{c}\right) / \Delta \phi\right)^{2}}
$$

where $\phi_{\mathrm{c}}$ is the parameter associated with the locus of the desired corner, and $\left(p_{0}+p_{c}\right)$ is its sharpness (see Fig. 6(b)). Similarly, we may make use of a "moving origin" to even out the parameterization density on the resulting curve (surface). Consider an origin that depends on $\phi$ (and $\theta$ in three dimensions). We employ Eq. (10) continuously,

$$
\begin{aligned}
r(\theta, \phi)=( & \sum_{i=1}^{N} H\left(\frac{\Delta(\theta, \phi) \cdot \mathbf{e}_{i}}{d_{i}-\Delta \mathbf{R}(\theta, \phi) \cdot \mathbf{e}_{i}}\right) \\
& \left.\times\left|\frac{\Delta(\theta, \phi) \cdot \mathbf{e}_{i}}{d_{i}-\Delta \mathbf{R}(\theta, \phi) \cdot \mathbf{e}_{i}}\right|^{p}\right)^{-1 / p}
\end{aligned}
$$

and also use Eq. (6) with the same parameterized origin, compensating the associated shifts. As shown in Figure 7, this yields two different representations of the identical curve (surface)-however, with different distributions of equidistant parameter points (lines).

Note that, to be meaningful, such a shifting origin should exhibit the same kind of basic properties as the parameter space. Specifically, it should be smooth and $2 \pi$ periodic in $\phi$, which suggests trigonometric functions in $\phi$ as the fundamental parameter in its construction.

So far, we have only considered the case of a limiting surface composed of flat facets. For some application it is necessary to consider more general, curved "facets" by replacing in Eq. (12) the flat-facet term by an arbitrary function

$$
\left|\frac{\Delta(\theta, \phi) \cdot \mathbf{e}_{i}}{d_{i}-\Delta \mathbf{R}(\theta, \phi) \cdot \mathbf{e}_{i}}\right| \rightarrow F_{i}(\theta, \phi)
$$
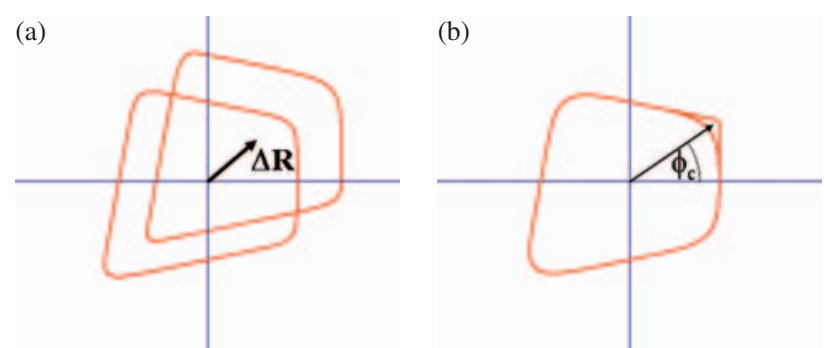

Fig. 6. (a) Relocating the origin by Eq. (10) to shift the entire curve. (b) Increasing the sharpness of individual corners with Eq. (11). 

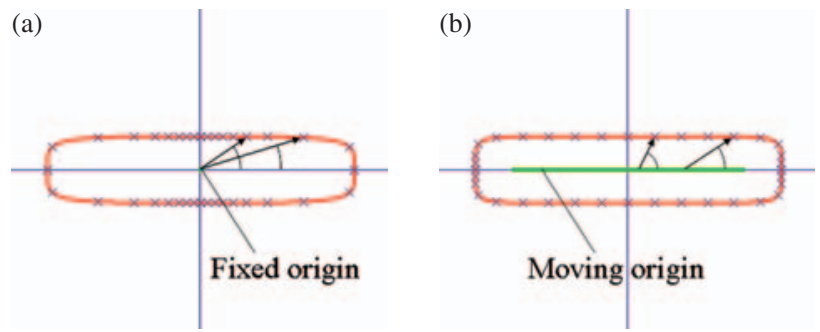

Fig. 7. Use of a moving origin for parameterization of a rectangular curve with aspect ratio 1:4 and corner rounding parameter $p=5$. (a) Global origin $\Delta \mathbf{R}(\phi)=\mathbf{0}$. (b) Moving origin $\Delta \mathbf{R}(\phi)=3 \cos (\phi) \mathbf{e}_{x}$.

Figure 8 presents an example, based on that of Figure 7, where the top facet description given by

$$
F_{i}(\theta, \phi)=\frac{\left|\Delta(\theta, \phi) \cdot \mathbf{e}_{i}\right|^{q}}{\left|d_{i}-\Delta \mathbf{R}(\theta, \phi) \cdot \mathbf{e}_{i}\right|}
$$

Depending on the parameter $q$, this generates a symmetric "dip" or "bump" in the center of of the modified facet.

\subsection{Generating $\nu$-Fold Rotationally Symmetric Curves}

As our final extension of the basic Eqs. $(6,7)$, we consider an option that utilizes the $2 \pi$-periodicity in $\phi$. Multiplying this parameter with a positive integer $\nu$, increases the periodicity without sacrificing continuity in the generated curve (surface),

$$
\begin{aligned}
r(\theta, \phi)=( & \sum_{i=1}^{N} H\left(\frac{\Delta(\theta, \nu \phi) \cdot \mathbf{e}_{i}}{d_{i}-\Delta \mathbf{R}(\theta, \phi) \cdot \mathbf{e}_{i}}\right) \\
& \left.\times\left|\frac{\Delta(\theta, \nu \phi) \cdot \mathbf{e}_{i}}{d_{i}-\Delta \mathbf{R}(\theta, \phi) \cdot \mathbf{e}_{i}}\right|^{p}\right)^{-1 / p \nu^{2}}
\end{aligned}
$$

An example is shown in Figure 9, where a three-fold rotationally symmetric shape is generated from a basic triangular shape by setting the distance of one facet to a near-zero value.

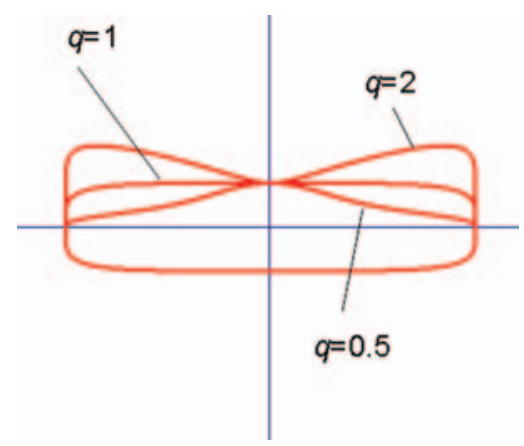

Fig. 8. Generation of curved surfaces. Here, the top facet is altered by using the flat-facet function $F_{i}(\phi, \theta)$ as indicated in Eq. (14) with different values for the convexity parameter $q$.

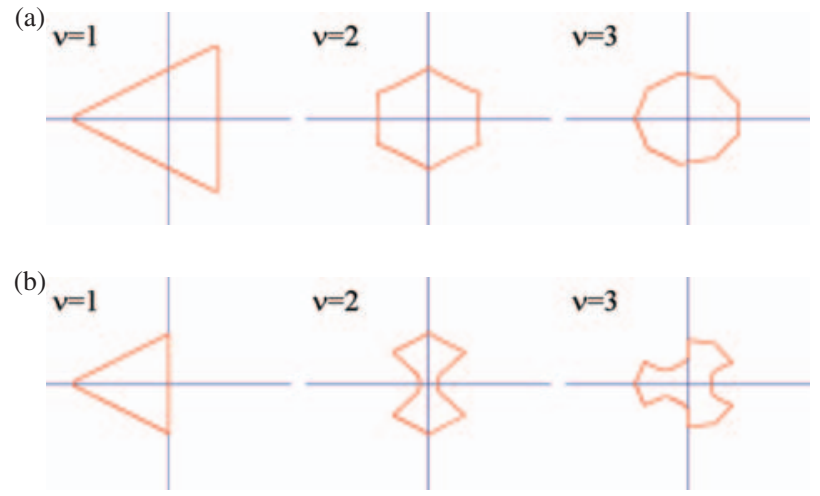

Fig. 9. Higher periodicity curves, generated with Eq. (15). (a) $N=3$, $d_{i}=1$. (b) The same as (a), but with $d_{1}=0.001$.

The additional factor of $\nu^{2}$ in the exponent of Eq. (15) is a reasonable approximant to render flat facets in the case of regular polygons. It may also be parameterized with $\phi$ (and $\theta$ ), of course, to control the local curvature exactly. The most interesting applications, however, may indeed be shapes that grossly deviate from regular polygons (which may be generated exactly with the help of Eq. (7), anyway). As is shown in Figure 10, starting from an elongated rectangular shape, one can generate quad- and hexapods.

\subsection{Three-Dimensional Surface Constructions}

All of the concepts demonstrated above for the twodimensional case apply also to three dimensions. Thus, a parameterization of arbitrary convex enclosures can be achieved in terms of the usual spherical angles $(0 \leq \phi 0 \leq$ $2 \pi, 0 \leq \theta \leq \pi)$. This offers an intuitive way to construct technologically or scientifically relevant objects. For instance, in the field of current nano-optical research, the vast majority of particle shapes encountered is covered. Unit cell symmetries and chemical growth processes typically enforce single and multiply twinned crystals, which are described as cuboids, platelets, rods, spheroids, etc. (Fig. 11).

An interesting challenge that remains is a more general control over non-convex structures. Being based on spherical coordinates, it should be possible to parameterize a rich variety of star-like domains. Based on the principle of a moving origin (in three-dimensions, it may move on a parameter surface), it might even be possible to generate $(\phi, \theta)$-parameterizations for more complex domain shapes.

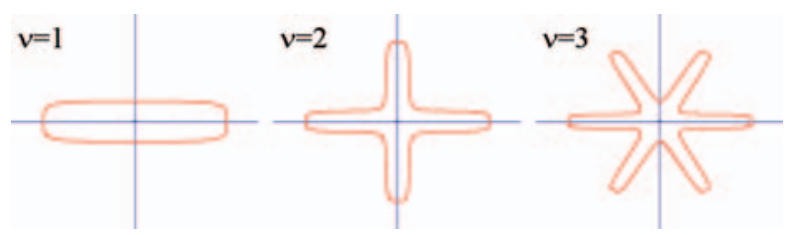

Fig. 10. Higher periodicity curves, generated with Eq. (15) without the additional factor $\nu^{2}$ in the exponent. 
(a)

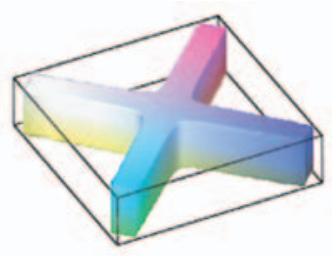

(d)

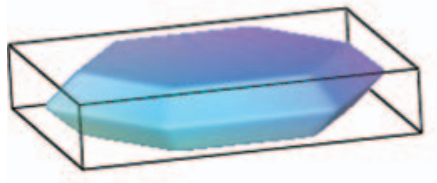

(b)

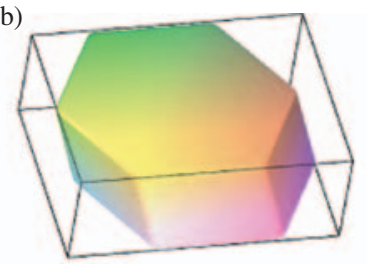

(e)

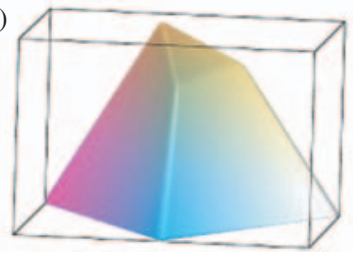

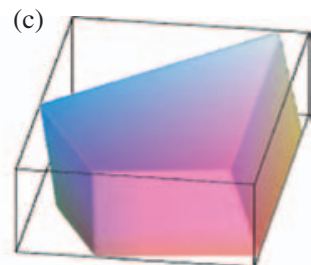

(f)

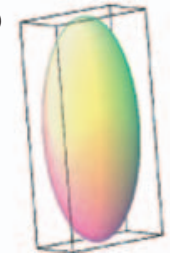

Fig. 11. Basic three-dimensional shapes created with Eqs. (6, 7). (a-c) Prisms generated from two-dimensional curves with side walls (a) straight-up, (b) slanted, and (c) individually controlled. (d) Hexagonal platelet with angled side walls. (e) Tetrahedron with tip cut away. (f) General ellipsoid.

$\mu=1 \mathrm{v}=1$
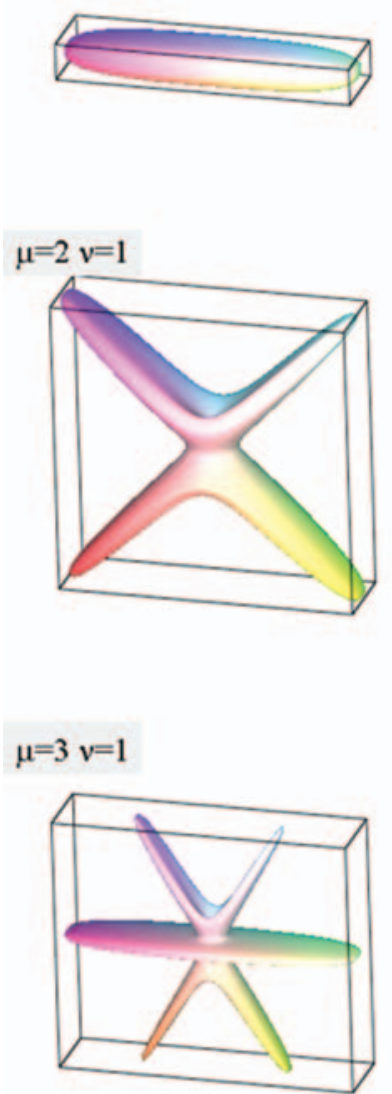

$\mu=1 \quad v=1.5$
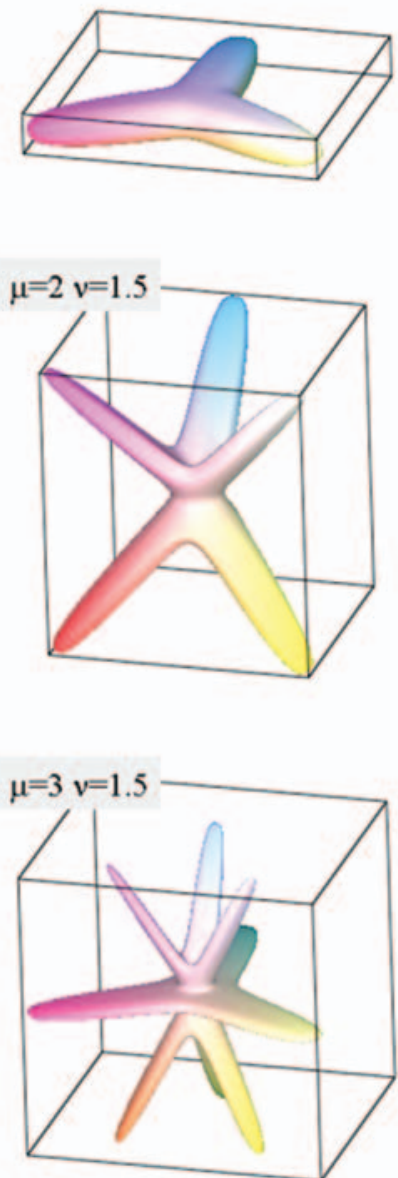

$\mu=1 v=2$
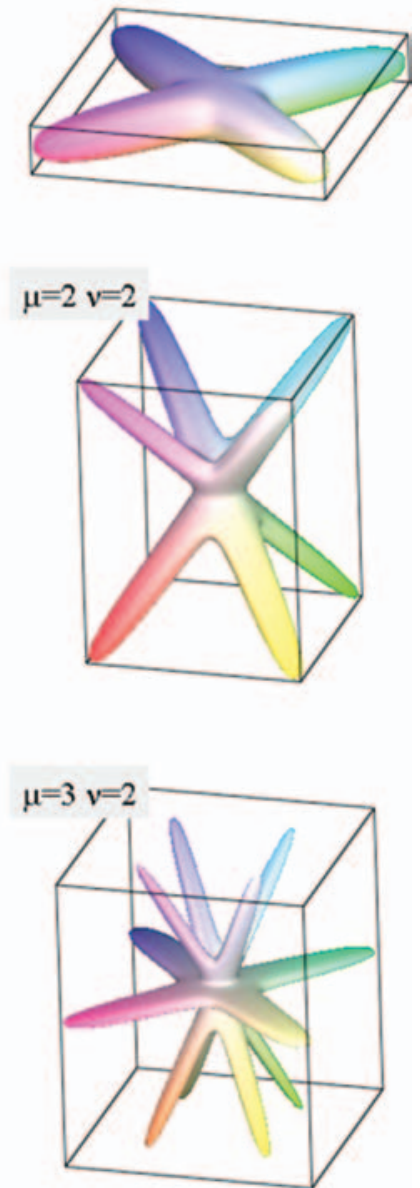

Fig. 12. The effect of symmetry order factors in the parameters for the example of a symmetric bar. Note that half-integer $\nu$-values yield closed surfaces only if the basic $(\mu=\nu=1)$ structure is symmetric. 
At this point we mainly have the symmetry extension discussed in Eq. (15) at our disposal. Its generalization to the three-dimensional case introduces a multiplier $\mu$ to the $\theta$-direction:

$$
\begin{aligned}
r(\theta, \phi)=\left(\sum_{i=1}^{N} H\left(\frac{\Delta(\mu \theta, \nu \phi) \cdot \mathbf{e}_{i}}{d_{i}-\Delta \mathbf{R}(\theta, \phi) \cdot \mathbf{e}_{i}}\right)\right. \\
\left.\quad \times\left|\frac{\Delta(\mu \theta, \nu \phi) \cdot \mathbf{e}_{i}}{d_{i}-\Delta \mathbf{R}(\theta, \phi) \cdot \mathbf{e}_{i}}\right|^{p}\right)^{-1 / p \nu^{2}}
\end{aligned}
$$

Figure 12 displays how, starting from a symmetric bar, a sequence of highly symmetric star-like structures are obtained. They do resemble some nano-structures that have been recently fabricated, however, not very closely. One would like to be able to control the orientation, length and shape of individual arms. This might be possible through continued parameterization of $\mu, \nu, p$-i.e., making them intricate functions of $\theta$ and $\phi$.

\subsection{Surface Generation from (Measured) Point Clouds}

The cases we have presented above may appear somewhat idealized, in that they were driven by the desire for an accurate, yet versatile representation of polyhedrons, which require only a small number of flat facets. It may be appreciated, though, that there is neither a principal limitation to the number of facets, nor their curvature (see Eq. (13) and Fig. 8). Both of these aspects suggest the possibility of construction of a general surface from a point cloud with this scheme. Figure 13 gives an example. Here, a cloud of $40 \times 40$ surface locations is used. The surface representation is generated without concern to any local properties of the cloud. Each point $i$ in the cloud is associated with one independent facet. Its distance $d_{i}$ is set to the length of the vector from the origin to the point, and the surface normal $\mathbf{e}_{i}$ is set to the direction of this vector. To achieve any concave surface at all, some modifications such as indicated in Eq. (14) are necessary. We use a globally constant exponent $q=20$ with Eq. (14), whose outward-pulling, concave effect is manually balanced with the inward-pulling, smoothing effect of a moderate exponent $p=10$ in Eq. (7).

From this particular example, a number of likely issues are identified that will help obtaining a better representation in the generic case: Independent knowledge of the surface normal direction in each point is highly desirable, instead of a priori assumptions or intricate analysis of the cloud. The value of $q$ should be made variable over the index $i$, as it depends on the local density of input points, as well as the local curvature to be achieved. Similarly, $p$ maybe localized in the vein of Eq. (11). Finally, we note that open surfaces are also directly attainable, using only sub intervals of the $(\phi, \theta)$ parameter space. In conjunction with mapping the origin location (Eq. (15)) and/or the
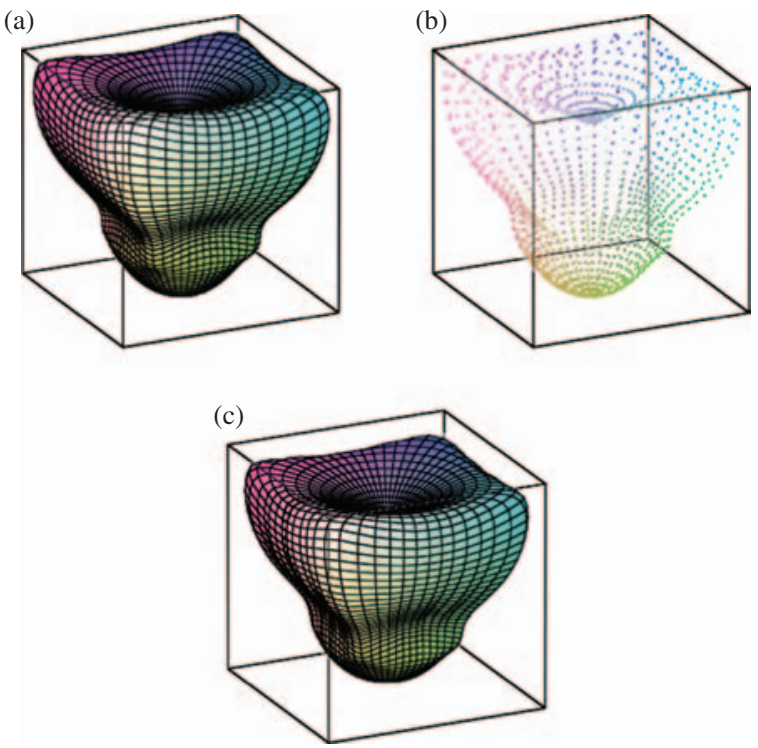

Fig. 13. Demonstration of the use of "smooth facet selection" to represent general surfaces. (a) The ideal surface to be represented. (b) Point cloud of surface locations used as input for generating a representation of the original surface. (c) Representation of (a) with Eqs. $(6,7,13)$.

local coordinate system, the present method may even be developed into an alternative to Fourier continuation, ${ }^{19,20}$ also yielding $C^{\infty}$-smooth surfaces essential for high-order numerical methods.

\section{SUMMARY}

We have presented a versatile approach to the $C^{\infty}$-smooth global parameterization of the surface of star-like domains. It is based on the "choosing" quality of the $p$-norm, which for $p \rightarrow \infty$ becomes the "maximum" norm-verbalizing this selection process explicitly. Here, the choice falls for given parameters $(\theta, \phi)$ on one of an arbitrary set of limiting facets, which can thus be oriented completely at will. The singular limiting facet-joints, i.e., edges and corners, can be given tunable sharpness.

This scheme is rather different from approaches based on tensor products or superquadrics. ${ }^{24,25}$ which nevertheless may be combined with our present approach, when convenient. For example, our two-dimensional representation may be used with the standard spherical tensor product to render straight cylindrical prisms or rotationally symmetric objects. We have demonstrated a number of different principles to extend the range of possible applications of the central Eqs. $(6,7)$. These functions execute fast and - as they are known analytically — can be used further to devise strategies for setting other quantities important to simulations. For example, gradients and surface normals may also be expressed analytically, and the local curvature may be used to define sampling density on the parameterized surface. 
Particular benefits of this modeling procedure are foreseen in the evaluation of optical properties of nano-objects. A close similarity of thermo-chemical growth processes with the morphing strategies of the present parameterization allows for a very intuitive and realistic rendering of their geometries. Beyond this, many useful applications are possible with essentially any boundary-type solver for differential and integral equations.

Acknowledgments: The author would like to acknowledge many fruitful discussions with Ludmila Raguin and Christian Hafner.

\section{References}

1. X. M. Lu, M. Rycenga, and S. E. Skrabalak, Annu. Rev. Phys. Chem. 60, 167 (2009).

2. T. K. Sau and A. L. Rogach, Adv. Mater. 22, 1781 (2010).

3. T. K. Sau, A. L. Rogach, and F. Jackel et al., Adv. Mater. 22, 1805 (2010).

4. R. Vogelgesang and A. Dmitriev, Analyst 135, 1175 (2010).

5. T. Zentgraf, J. Dorfmuller, and C. Rockstuhl et al., Opt. Lett. 33, 848 (2008).

6. R. Esteban, R. Vogelgesang, and J. Dorfmuller et al., Nano Lett. 8, 3155 (2008).

7. J. Dorfmuller, R. Vogelgesang, and R. T. Weitz et al., Nano Lett. 9, 2372 (2009).

8. J. Dorfmuller, R. Vogelgesang, and W. Khunsin et al., Nano Lett. 10, 3596 (2010).
9. U. Hohenester and J. Krenn, Physical Review B 72 (2005).

10. D. R. Fredkin and I. D. Mayergoyz, Phys. Rev. Lett. 91 (2003).

11. V. Myroshnychenko, E. Carb-Argibay, and I. Pastoriza-Santos et al., Adv. Mater. 20, 4288 (2008).

12. V. Myroshnychenko, J. Rodriguez-Fernandez, and I. PastorizaSantos et al., Chem. Soc. Rev. 37, 1792 (2008).

13. R. Esteban, R. Vogelgesang, and K. Kern, Physical Review B 75 (2007).

14. R. Esteban, R. Vogelgesang, and K. Kern, Opt. Express 17, 2518 (2009).

15. J. T. Katsikadelis, Boundary Elements: Theory and Applications, Elsevier Science Ltd., Oxford (2002).

16. H. Ammari, Modeling and Computations in Electromagnetics, Springer, Berlin, Heidelberg, New York (2007).

17. W. C. Gibson, The Method of Moments in Electromagnetics, Chapman and Hall/CRC, Boca Raton, FL (2008).

18. W. C. Chew, M. S. Tong, and B. Hu, Integral Equation Methods for Eletromagnetics and Elastic Waves, Morgan and Claypool (2009).

19. O. P. Bruno, Y. Han, and M. M. Pohlman, Journal of Computational Physics 227, 1094 (2007).

20. O. P. Bruno, Topics in Computational Wave Propagation, edited by M. Ainsworth, P. Davies, D. Duncan, P. Martin, and B. Rynne Springer, Berlin, Heidelberg, New York (2003).

21. V. Rokhlin and M. Tygert, SIAM Journal on Scientific Computing 27, 1903 (2006).

22. M. Tygert, Journal of Computational Physics 227, 4260 (2008).

23. M. Tygert, Journal of Computational Physics 229, 6181 (2010).

24. D. Terzopoulos and D. Metaxas, IEEE Transactions on Pattern Analysis and Machine Intelligence 13, 703 (1991).

25. R. Dietz, J. Hoschek, and B. Juttler, Computer Aided Geometric Design 10, 211 (1993).

Received: 23 September 2010. Accepted: 28 October 2010. 